\title{
EL SALARIO BASE DE COTIZACIÓN Y SUS IMPLICACIONES EN EL PAGO DE LAS PRESTACIONES EN DINERO DEL SEGURO DE RIESGO DE TRABAJO
}

\author{
THE CONTRIBUTION WAGE BASE AND ITS \\ IMPLICATIONS IN THE PAYMENT OF THE CASH \\ BENEFITS OF OCCUPATIONAL HAZARD
}

\author{
Dr. Edgar Tapia Lara ${ }^{a}$ \\ Dr. Salvador Sánchez Ruanova ${ }^{b}$ \\ Dr. Sergio Gabriel Ordoñez Sanchez ${ }^{c}$ \\ Lic. Alma Lazacano Bonilla ${ }^{d}$
}

\begin{abstract}
a Benemérita Universidad Autónoma de Puebla Facultad de Contaduría Pública, etapialara@yahoo.com.mx

b Benemérita Universidad Autónoma de Puebla Facultad de Contaduría Pública, cpcruanova@hotmail.com

c) Benemérita Universidad Autónoma de Puebla Facultad de Contaduría Pública, sergio.buap@gmail.com

d Benemérita Universidad Autónoma de Puebla Facultad de Contaduría Pública, al_greg@outlook.com
\end{abstract}

\section{RESUMEN}

El Salario Base Cotización juega un papel fundamental para la determinación de las Cuotas Obreras Patronales, siendo esta la razón por la que los patrones en la búsqueda de disminuir su carga tributaria en materia de aportaciones de seguridad social, utilizan para el pago de sus empleados conceptos excluyentes para la determinación del salario base de cotización, con fundamento en el artículo 27 de Ley de la ley del seguro social, lo anterior se encuentra dentro de la legalidad si se cumplen los requisitos establecidos en dicho artículo, para el pago de cada prestación que se otorgue al trabajador, sin embargo, esta situación provoca una disminución directa en el monto pagado a los trabajadores respecto a las prestaciones en dinero que otorga el seguro de riesgos de trabajo del régimen obligatorio, ya que son calculadas sobre el salario base de cotización que estuviese cotizando el trabajador al momento de sufrir el riesgo de trabajo. Razón por la cual surge esta investigación, teniendo objeto identificar, cuantificar y medir las implicaciones que tiene el Salario Base de Cotización al momento del pago de las prestaciones en dinero del seguro de riesgos de trabajo, al considerarse como base para el pago de las cuotas obrero-patronales y para el pago de las prestaciones en dinero del seguro de riesgos de trabajo. 
Palabras Clave: Salario Base de Cotización; Riesgo de Trabajo; Prestaciones en Dinero y Trabajador.

\begin{abstract}
The contribution wage base has a fundamental role in the determination of the employee -employer quota. This is the reason why the institutions look to reduce their tax burden in social security contributions. They use excluding concepts to determinate the contribution wage base for the employee's payment, based on article 27 of the Social Security Act, the above is legal if the requirements established in that article are fulfilled, for the payment of each benefit granted to the employee, however, this situation causes a direct decrease in the amount paid to the employee's with respect to the cash benefits granted by the occupational hazard of the compulsory scheme, since they are calculated by the contribution wage base that the employee was quoting at the time of suffer the occupational hazard. This is the reason why this investigation arises, which aims to identify, quantify and measure the implications of the contribution wage base at the time of the payment of the cash benefits of occupational hazard, when being considered as a base for the payment of the employee-employer quota and of the payment of the benefits in cash of the occupational risk insurance.
\end{abstract}

KEY WORDS: Contribution wage base; occupational hazard, cash benefits; employee.

\title{
I. INTRODUCCIÓN
}

La ley del seguro social en su título II determina los seguros del régimen obligatorio, formando parte de estos, el seguro de riesgos de trabajo, el cual establece para los asegurados en vida, así como para sus beneficiarios en caso de su muerte al sufrir el riesgo de trabajo, prestaciones en dinero, las cuales deberán ser pagadas sobre el salario base de cotización que tuviese el trabajador al momento de presentarse el accidente de trabajo, o bien sobre el promedio del salario base de cotización de las ultimas cincuenta y dos semanas tratándose de enfermedad profesional, dicho salario base de cotización es calculado considerando las prestaciones que reciba el trabajador, derivadas de la relación laboral y considerando las disposiciones que esta ley estipula en el capítulo II, del título II ya comentado, sin perder de vista aquellas que hace referencia el artículo 27 de la ley del seguro social en sus fracciones de la I a la IX, respecto a las disposiciones de ley federal del trabajo.

\section{PLANTEAMIENTO DEL PROBLEMA}

El problema de esta investigación radica en el hecho de considerar para el pago de las prestaciones en dinero del seguro de riesgos de trabajo, el salario base de cotización ya que este considera los excluyentes permitidos y normados en términos del artículo 27 de

Tapia, E., Sánchez, S., Ordoñez, S.G. \& Lazcano, A.

El salario base de cotización y sus implicaciones en el pago de las prestaciones en dinero del seguro de riesgo de trabajo 
la ley del seguro social, lo cual implica una disminución real en el pago al trabajador de cualquiera de las prestaciones en dinero a las que tiene derecho en términos seguro de riesgos de trabajo (capítulo III, sección III de la ley del seguro social).

\section{OBJETIVOS}

\section{Objetivo general:}

- Demostrar la disminución real que tiene el trabajador, respecto al pago que recibe de cualquier prestación en dinero del seguro de riesgos de trabajo.

\section{Objetivos específicos:}

- Lograr concientizar a patrones y trabajadores por medio de los resultados de esta investigación, sobre esta disminución real del pago que tiene el trabajador.

- Buscar que, a través de los resultados obtenidos en esta investigación, se pueda llegar a un cambio respecto a la base para la determinación del pago de las prestaciones en dinero del seguro de riesgos de trabajo.

\section{MARCO TEÓRICO-CONCEPTUAL}

\section{IV.I Contexto General}

La Ley del seguro social en su artículo 6, señala que el seguro social comprende el régimen obligatorio y el régimen voluntario, siendo sujetos del régimen obligatorio en términos del artículo 12 los patrones y los trabajadores al configurase la relación de laboral en términos del artículo 21 de la ley federal de trabajo, razón por la cual en términos del artículo 12 y 15 fracción III de la ley del seguro social, los patrones y trabajadores están obligados a cubrir las cuotas obrero patronales de los seguros del régimen obligatorio que son el de riesgos de trabajo, enfermedades y maternidad, invalidez y vida, retiro, cesantía en edad avanzada y vejez y guarderías y prestaciones sociales (IMSS, 2018) (Cruz, 2014).

\section{IV.II Salario Base de Cotización}

Los patrones y trabajadores para el cumplimiento de esta obligación consideran como base para el pago de las cuotas obrero-patronales el Salario Base de Cotización, siendo fundamental para su determinación el artículo 27 de la ley del seguro social el cual señala en su primer párrafo el salario base de cotización se integra con:

1. Los pagos hechos en efectivo por cuota diaria.

2. Gratificaciones.

3. Percepciones.

Tapia, E., Sánchez, S., Ordoñez, S.G. \& Lazcano, A.

El salario base de cotización y sus implicaciones en el pago de las prestaciones en dinero del seguro de riesgo de 138 trabajo 
4. Alimentación.

5. Habitación.

6. Primas.

7. Comisiones.

8. Prestaciones en especie.

9. Y cualquiera otra cantidad o prestación que se entregue al trabajador por su trabajo.

Sin embargo, en el mismo párrafo contempla que se excluyen como integrantes del salario base de cotización, dada su naturaleza, los siguientes conceptos:

I. Los instrumentos de trabajo tales como herramientas, ropa y otros similares.

II. El Fondo de ahorro, cuando se integre por una aportación igual por parte del trabajador y de la empresa sin importar su periodicidad, ya que si se constituye en forma diversa o puede el trabajador retirarlo más de dos veces al año integrará salario base de cotización; tampoco se tomarán en cuenta las cantidades otorgadas por el patrón para fines sociales de carácter sindical.

III. Las aportaciones adicionales que el patrón convenga otorgar a favor de sus trabajadores por concepto de cuotas del seguro de retiro, cesantía en edad avanzada y vejez.

IV. Las cuotas que en términos de esta Ley le corresponde cubrir al patrón, las aportaciones al Instituto del Fondo Nacional de la Vivienda para los Trabajadores, y las participaciones en las utilidades de la empresa.

V. La alimentación y la habitación cuando se entreguen en forma onerosa a los trabajadores; se entiende que son onerosas estas prestaciones cuando el trabajador pague por cada una de ellas, como mínimo, el veinte por ciento del salario mínimo general diario que rija en el Distrito Federal.

Por su parte el artículo 32 de la ley del seguro social complementa la regulación de éstos dos conceptos, citando que cuando se entregue al trabajador alimentación y habitación en forma gratuita se integrará como parte del salario base de cotización un veinticinco por ciento sobre su cuota diaria por cada concepto precisando en el caso de alimentación que se aumentará la cuota diaria del trabajador al presentarse este supuesto en un ocho punto treinta y tres por ciento por cada alimento por lo que

Tapia, E., Sánchez, S., Ordoñez, S.G. \& Lazcano, A.

El salario base de cotización y sus implicaciones en el pago de las prestaciones en dinero del seguro de riesgo de trabajo 
solo se considera el veinticinco porciento cuando se le otorguen los tres alimentos al trabajador.

Para cuantificar si el importe mínimo a que hace referencia esta fracción para que el otorgamiento de dichos conceptos se considere oneroso se toma como base la UMA (Unidad de medida de actualización) que este vigente al momento de otorgar dichas prestaciones.

VI. Las despensas en especie o en dinero, siempre y cuando su importe no rebase el cuarenta por ciento del salario mínimo general diario vigente en el Distrito Federal.

El monto del cuarenta por ciento a que hace referencia esta fracción se calcula sobre UMA (Unidad de medida de actualización) que esté vigente al momento de otorgar dichas prestaciones.

VII. Los premios por asistencia y puntualidad, siempre que el importe de cada uno de estos conceptos no rebase el diez por ciento del salario base de cotización.

Para el cálculo del diez por ciento a que hace referencia esta fracción se tendrá que llevar a cabo una integración parcial considerando todas las prestaciones que tenga el trabajador con excepción de estos dos conceptos y dicho resultado será al que se le aplique dicho porcentaje siendo el resultado que se compara con el importe diario de dichas prestaciones para efectos de saber si se integran o se excluyen del salario base de cotización.

VIII. Las cantidades aportadas para fines sociales, considerándose como tales las entregadas para constituir fondos de algún plan de pensiones establecido por el patrón o derivado de contratación colectiva.

Los planes de pensiones serán sólo los que reúnan los requisitos que establezca la Comisión Nacional del Sistema de Ahorro para el Retiro.

IX. El tiempo extraordinario dentro de los márgenes señalados en la Ley Federal del Trabajo.

Para tal efecto la ley federal del trabajo en su artículo 66 establece que podrá prolongarse la jornada de trabajo por circunstancias extraordinarias, sin exceder nunca de tres horas diarias ni de tres veces en una semana siendo este limite al que hace referencia esta fracción, de igual forma el artículo 67 de la misma ley establece que el pago de las horas de trabajo extraordinario se pagarán con un ciento por ciento más del salario que corresponda a las horas de la jornada y el excedente de nueve horas a la semana, obliga al patrón a pagar al trabajador el tiempo excedente con un

Tapia, E., Sánchez, S., Ordoñez, S.G. \& Lazcano, A.

El salario base de cotización y sus implicaciones en el pago de las prestaciones en dinero del seguro de riesgo de 140 trabajo 
doscientos por ciento más del salario que corresponda a las horas de la jornada esto en términos del artículo 69 de la ley federal del trabajo (Olguín, 2017).

Estableciendo como un requisito adicional que dichos conceptos deberán estar debidamente registrados en la contabilidad del patrón.

En el caso de de premios de puntualidad y asistencia, despensa y tiempo extraordinario cuando su importe rebase el importe establecido como excluyente, solo se integrará al salario base de cotización el excedente (IMSS, 2018) (Gutierrez, 2018).

\section{IV.III Pago de Cuotas Obrero-Patronales}

En términos del artículo 29 fracción I de la ley del seguro social, el pago de las cuotas obrero-patronales se determina por mes natural, teniendo como fecha límite de pago el día 17 del mes inmediato siguiente al que corresponda el pago, esto con base al artículo 36 de la ley del seguro social, el patrón estará obligado a determinar y pagar el importe de las cuotas a su cargo y fungir como retenedor para efectos del entero de las cuotas obrero patronales en términos de los artículos 15 fracción III y el 39 de la ley del seguro social, excepto que se trate de trabajadores que estén cotizando con salario mínimo establecido en artículo 28 y considerando lo dispuesto en el artículo 36 de la ley del seguro social que obliga al patrón a pagar íntegramente la cuota.

Para efectos del pago de cuotas obrero-patronales el patrón podrá utilizar como forma de pago efectivo, cheques certificados o de caja, así como las transferencias electrónicas de fondos y tarjetas de crédito o de débito expedidas por instituciones de crédito o bien mediante la aplicación de notas de crédito que emita el Instituto Mexicano del Seguro Social (IMSS, 2018) (Cruz, 2014).

\section{IV.IV Seguro de Riesgos de Trabajo}

El artículo 41 de la ley del seguro social define como riesgos de trabajo los accidentes y enfermedades a los que están expuestos los trabajadores en ejercicio o con motivo del trabajo.

Entendiéndose en términos del artículo 42 de la ley del seguro social como accidente de trabajo:

"Toda lesión orgánica o perturbación funcional, inmediata o posterior; o la muerte, producida repentinamente en ejercicio, o con motivo del trabajo, cualquiera que sea el

Tapia, E., Sánchez, S., Ordoñez, S.G. \& Lazcano, A.

El salario base de cotización y sus implicaciones en el pago de las prestaciones en dinero del seguro de riesgo de trabajo 
lugar y el tiempo en que dicho trabajo se preste" (IMSS, 2018) y en términos del artículo 43 de la ley del seguro social como enfermedad profesional:

"Todo estado patológico derivado de la acción continua de una causa que tenga su origen o motivo en el trabajo, o en el medio en que el trabajador se vea obligado a prestar sus servicios" (IMSS, 2018).

\section{IV.V Consecuencias del Riesgo de Trabajo}

En términos del artículo 56 de la ley del Seguro Social las consecuencias de un riesgo de trabajo son:

I. Incapacidad temporal.

II. Incapacidad permanente parcial.

III. Incapacidad permanente total.

IV. Muerte.

\section{IV.VI Prestaciones en Dinero}

En términos del artículo 58 de la ley del seguro social los trabajadores tienen derecho al pago de las siguientes las prestaciones en dinero:

\section{- Incapacidad Temporal}

Si derivado del riesgo de trabajo el trabajador se incapacita para el desempeño de sus labores recibirá mientras dure la inhabilitación, el cien por ciento del salario base de cotización con el que estuviese cotizando al momento de ocurrir el accidente de trabajo o se dictamine la enfermedad profesional, el pago de éste subsidio lo realizará el Instituto Mexicano del Seguro Social durante el periodo que el trabajador no se encuentre capacitado para trabajar o bien hasta que sea declarada por el mismo instituto la incapacidad permanente parcial o total, lo cual deberá suceder en un plazo no mayor a cincuenta y dos semanas, tiempo en el cual el trabajador recibirá atención médica como consecuencia del accidente o enfermedad profesional, sin perjuicio de que una vez determinada la incapacidad permanente parcial o total que corresponda, continúe su atención o rehabilitación(IMSS, 2018) (Públicos, Acuerdos Relevantes del Consejo Tecnico del IMSS, 2015).

Tapia, E., Sánchez, S., Ordoñez, S.G. \& Lazcano, A.

El salario base de cotización y sus implicaciones en el pago de las prestaciones en dinero del seguro de riesgo de 142 trabajo 


\section{- Incapacidad Permanente Parcial}

Si como consecuencia del riesgo de trabajo al trabajador se le otorga un dictamen por incapacidad permanente parcial con una valuación definitiva entre un cero punto cero uno y veinticinco por ciento como máximo, se pagará al trabajador una indemnización global equivalente a cinco anualidades de la pensión que le hubiese correspondido, en sustitución de la pensión definitiva por este concepto.

Cuando la valuación exceda del veinticinco por ciento, pero no del cincuenta por ciento el trabajador podrá elegir entre cobrar dicha indemnización o bien disfrutar de la pensión por incapacidad permanente parcial que le corresponda (IMSS, 2018) (Cruz, 2014).

\section{- Incapacidad Permanente Total}

Si la consecuencia del riesgo de trabajo es un dictamen por incapacidad permanente total, el trabajador recibirá una pensión mensual definitiva equivalente al setenta por ciento del salario base de cotización que estuviese cotizando en el momento de ocurrir el accidente de trabajo.

En el caso de enfermedad profesional, se calculará con el promedio del salario base de cotización de las cincuenta y dos últimas semanas o las que tuviere el trabajador desde su fecha de ingreso a la fecha del dictamen de la incapacidad permanente total.

El monto de la valuación de la incapacidad permanente parcial y total se determinará con base a la tabla de valuaciones contenida en la Ley Federal de Trabajo, el por ciento de la incapacidad se fijará entre el máximo y el mínimo establecidos en dicha tabla, teniendo en cuenta la edad del trabajador, la importancia de la incapacidad, si ésta es absoluta para el ejercicio de su profesión, aun cuando quede habilitado para dedicarse a otra, o que simplemente hayan disminuido sus aptitudes para el desempeño de la misma o para ejercer actividades remuneradas semejantes a su profesión u oficio (IMSS, 2018) (Cruz, 2014).

\section{- Aguinaldo Anual}

El trabajador que este pensionado por incapacidad permanente total o parcial con una valuación superior al cincuenta por ciento, recibirá adicional a su pensión en el mes de diciembre, un aguinaldo anual equivalente a quince días del importe de la pensión que perciban (IMSS, 2018) (Cruz, 2014).

Tapia, E., Sánchez, S., Ordoñez, S.G. \& Lazcano, A.

El salario base de cotización y sus implicaciones en el pago de las prestaciones en dinero del seguro de riesgo de trabajo 


\section{- Pensiones a la muerte del trabajador}

El artículo 64 de la ley del seguro social establece las prestaciones en dinero que tendrán derecho los beneficiarios del pensionado por riesgo de trabajo a su muerte.

\section{- Gastos de Funeral}

La familia del trabajador fallecido recibirá una cantidad igual a sesenta días de salario mínimo general que rija en el Distrito Federal en la fecha de fallecimiento del asegurado.

Es importante mencionar que para el cálculo actual de los gastos de funeral se toma como base la UMA (Unidad de Medida de Actualización) vigente al momento del pago.

El pago se hará a la persona preferentemente familiar del trabajador fallecido por riesgo de trabajo, que presente copia del acta de defunción y la cuenta original de los gastos de funeral (IMSS, 2018) (social, 2008) (Gutierrez F. J., 2018).

\section{- Pensión de Viudez}

La viuda (esposa o concubina) del trabajador recibirá una pensión equivalente al cuarenta por ciento de la pensión por incapacidad permanente total o parcial que recibía el trabajador en vida o aquella que le hubiese correspondido por incapacidad permanente total en caso de no haber fallecido como consecuencia del riesgo de trabajo, dicha pensión de viudez también se le pagará al viudo (esposo o concubinario) que hubiera dependido económicamente del trabajador fallecido. Es importante destacar que el importe de esta pensión no podrá ser inferior a la cuantía mínima que corresponda a la pensión de viudez del seguro de invalidez y vida.

En el mes de diciembre adicional al pago de su pensión recibirán un aguinaldo anual equivalente a quince días de la pensión que percibían.

Para que la concubina o concubinario puedan recibir la pensión deben cumplir los siguientes requisitos:

1. Vivir como si fuera esposo o esposa del trabajador fallecido por riesgo de trabajo durante los cinco años que precedieron inmediatamente a su muerte $o$ haber tenido hijos.

2. Haber permanecido libres de matrimonio durante el concubinato.

Si al morir el asegurado tenía varias concubinas, ninguna de ellas gozará de pensión.

Tapia, E., Sánchez, S., Ordoñez, S.G. \& Lazcano, A.

El salario base de cotización y sus implicaciones en el pago de las prestaciones en dinero del seguro de riesgo de 144 trabajo 
El derecho al pago de la pensión de viudez se realizará mientras la viuda o viudo no contraiga nuevas nupcias o entre en concubinato esto términos del artículo 66 de la ley del seguro social.

Cuando se pierda el derecho al pago de esta pensión la viuda o viudo recibirán un finiquito equivalente a tres anualidades de la pensión que percibían (IMSS, 2018) (social, 2008) (Gutierrez F. J., 2018).

\section{- Pensión de Orfandad para Discapacitados}

Tendrá derecho a esta pensión cada uno de los huérfanos que lo sean de padre o madre, que se encuentren totalmente incapacitados, los cuales recibirán una pensión equivalente al veinte por ciento de la que hubiese correspondido al trabajador fallecido tratándose de incapacidad permanente total.

En caso de fallecimiento del otro progenitor el porcentaje de esta pensión será del 30\% y en caso de que con posterioridad fallezca el otro progenitor el porcentaje pasa del $20 \%$ al $30 \%$, el pago de esta pensión se extinguirá cuando el huérfano recupere su capacidad para el trabajo en este caso el huérfano recibirá un finiquito equivalente a tres mensualidades de pensión que percibía (IMSS, 2018) (Gutierrez F. J., 2018).

\section{- Pensión de Orfandad para menores de 16 años}

Cada uno de los hijos del trabajador fallecido por riesgo de trabajo que lo sean de padre o madre, menores de dieciséis años, recibirán una pensión equivalente al veinte por ciento de la que hubiera correspondido al asegurado tratándose de incapacidad permanente total. Esta pensión se extinguirá cuando el huérfano cumpla dieciséis años, sin embargo, deberá extenderse hasta una edad máxima de veinticinco años, cuando se encuentren estudiando en planteles del sistema educativo nacional, tomando en consideración, las condiciones económicas, familiares y personales del beneficiario y siempre que no sea sujeto del régimen obligatorio.

El porcentaje de esta pensión se incrementará cuando fallezca el otro progenitor del veinte por ciento al treinta por ciento, pero si al fallecer el asegurado se configura la doble orfandad se le otorga el treinta por ciento de la pensión que correspondió al asegurado en vida en caso de incapacidad permanente total y de manera adicional en el mes de diciembre recibirán un equivalente a 15 días de la pensión que reciben por concepto de aguinaldo anual. Al perder el derecho a la pensión recibirán un finiquito equivalente a tres mensualidades de la pensión que recibían (IMSS, 2018) (Gutierrez F. J., 2018).

Tapia, E., Sánchez, S., Ordoñez, S.G. \& Lazcano, A.

El salario base de cotización y sus implicaciones en el pago de las prestaciones en dinero del seguro de riesgo de trabajo 


\section{- Pensión de Ascendientes}

El artículo 66 de la ley del seguro social estipula que solo a falta de viuda o viudo, huérfanos, concubina o concubinario con derecho a pensión, se le otorga a cada uno de los padres que dependían económicamente del trabajador fallecido, una pensión equivalente al veinte por ciento de la pensión que le correspondía al asegurado, en el caso de incapacidad permanente total, esta pensión se extinguirá a la muerte de los beneficiarios (IMSS, 2018) (Gutierrez F. J., 2018).

\section{- Incremento de las Pensiones}

La cuantía de las pensiones por incapacidad permanente será actualizada anualmente en el mes de febrero, conforme al Índice Nacional de Precios al Consumidor correspondiente al año calendario anterior, en esta misma proporción serán incrementadas las pensiones de viudez, orfandad y ascendientes del asegurado por riesgos de trabajo (IMSS, 2018) (Gutierrez F. J., 2018).

\section{METODOLOGÍA Y TÉCNICAS EMPLEADAS}

La investigación se efectuó considerando una muestra de 100 trabajadores de empresas que realizan diferentes actividades, con la finalidad de poder comparar a trabajadores con diferentes prestaciones y de esta forma medir las implicaciones del salario base de cotización en el pago de las prestaciones en dinero del seguro de riesgos de trabajo.

\section{I. Método}

En la presente investigación se utilizó un método mixto a través del cual se abordó la información recabada de lo general a lo específico, permitiendo con esto resultados reales y susceptibles de su medición y cuantificación.

\section{V.II. Enfoque}

Esta investigación se realizó de manera cualitativa y cuantitativa ya que en primera instancia se analizó de manera general el seguro de riesgo de trabajo y las prestaciones de dinero que tiene derecho el trabajador, para posteriormente llevar a cabo una cuantificación de cada trabajador respecto al pago de dichas prestaciones y de esta manera identificar la disminución que se genera entre uno y otro trabajador dependiendo si cuenta o no con prestaciones excluyentes del salario base de cotización.

Tapia, E., Sánchez, S., Ordoñez, S.G. \& Lazcano, A.

El salario base de cotización y sus implicaciones en el pago de las prestaciones en dinero del seguro de riesgo de 146 trabajo 


\section{V.III. Tipo de investigación}

El presente trabajo de investigación se realizó de manera descriptiva, ya que al analizar la información proporcionada por los trabajadores de la muestra seleccionada se obtuvieron resultados reales y concretos.

\section{V.IV. Alcance}

El alcance de la investigación se llevó a cabo considerando una muestra de 100 trabajadores de empresas que otorgan a sus trabajadores diferentes prestaciones derivadas de la relación laboral.

\section{V. Ámbito Geográfico}

Esta investigación se realizó en la ciudad de Puebla, pero considerando el marco normativo aplicable en toda la república mexicana.

\section{V.IV. Investigación Documental}

La investigación documental se realizó mediante la consulta de fuentes como son: libros, tutoriales, simuladores de cálculo, direcciones electrónicas, revistas, leyes aplicables, acuerdos del consejo técnico y criterios normativos emitidos por el consejo técnico del Instituto Mexicano del Seguro Social. Lo cual en su conjunto permitió construir el marco teórico del presente trabajo de investigación.

\section{V.VII. Investigación de Campo}

El trabajo de campo se realizó con el análisis de la información proporcionada por la muestra de 100 trabajadores, los cuales fueron seleccionados con la finalidad de comparar la determinación del pago de las prestaciones en dinero del seguro de riesgos de trabajo, con base a la normatividad aplicable.

\section{RESULTADOS}

Se obtuvo un resultado contundente ya que los cálculos demuestran que el hecho de utilizar conceptos excluyentes del salario base de cotización tiene una disminución directa en el monto del pago de las prestaciones en dinero del seguro de riesgos de trabajo.

\section{VI.I. Resultados}

Tapia, E., Sánchez, S., Ordoñez, S.G. \& Lazcano, A.

El salario base de cotización y sus implicaciones en el pago de las prestaciones en dinero del seguro de riesgo de trabajo 
- El hecho de que las empresas otorguen conceptos excluyentes del salario base de cotización a sus trabajadores influye directamente en el monto que pagara por concepto de prestaciones en dinero del seguro de riesgos de trabajo.

- La disminución en el monto del pago de las prestaciones en dinero va directamente relacionada con las prestaciones que el trabajador reciba y que en términos del artículo 27 de la ley del seguro social que se excluyan del salario base de cotización.

- El hecho de que el patrón pague a sus trabajadores utilizando conceptos excluyentes del salario base de cotización, no implica que su determinación se llevó a cabo fuera del marco legal.

\section{VI.II. Datos:}

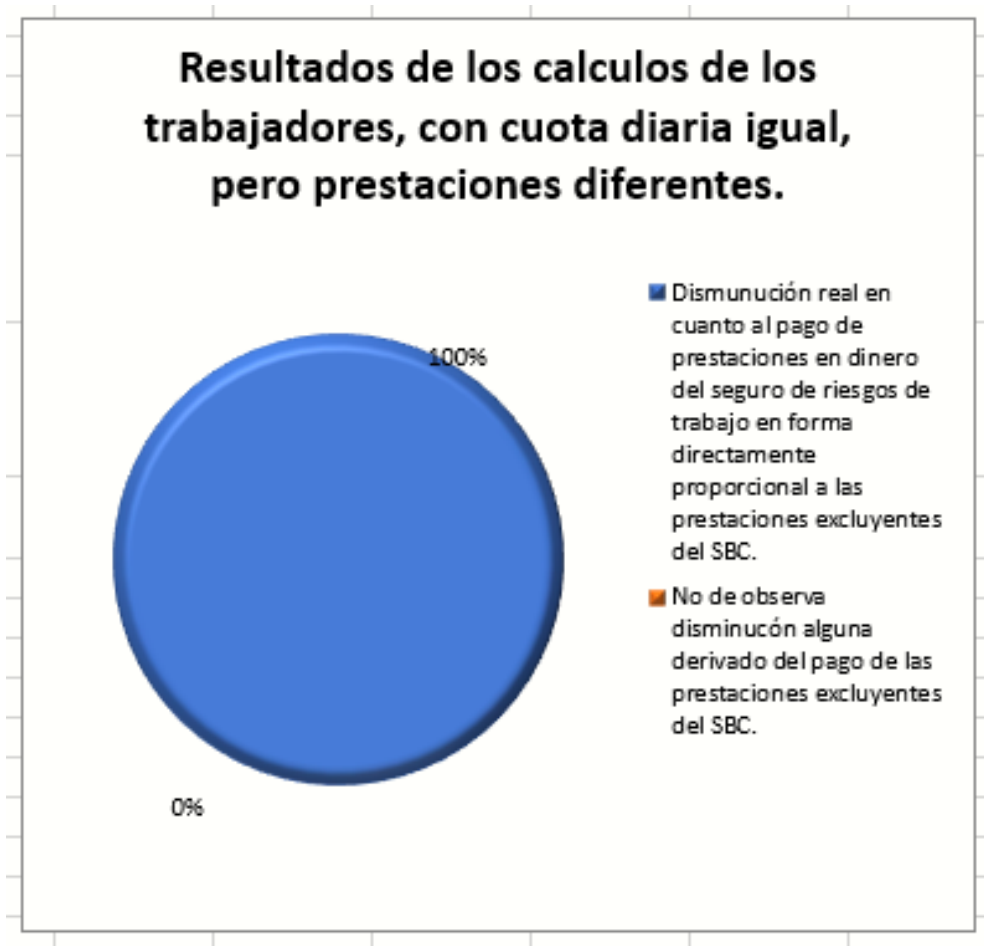

Figura 1. Resultados de los calculos de los trabajadores. fuente : Elaboración propia (2019)

Tapia, E., Sánchez, S., Ordoñez, S.G. \& Lazcano, A. 
TABLA 1

Resumen de los cálculos realizados con la muestra de trabajadores

\section{Resumen de los calculos realizados con la muestra de trabajadores}

Concepto

Salario Mensual $\$ 5,000.00$

Cuota Diaria

Aguinaldo 15 dias

Importe Diario

Prima Vacacional (Primer Año)

Importe Diario

Fondo de Ahorro Mensual $\$ 500.00$

(Cumple con los requistos del Art. 27 LSS)

Patrón

Premios de Puntualidad Mensual $\$ 550.00$

Importe Diario

Premios de Asistencia Mensual $\$ 1,100.00$

Importe Diario

Despensa Mensual \$1,000.00

Importe Diario

Ayuda para Renta de Casa Mensual $\$ 3,000.00$

(Cumple con los requistos del Art. 27 LSS)

Importe Diario

Ayuda para Alimentación (3 Alimentos) $\$ 3,000.00$

(Cumple con los requistos del Art. 27 LSS)

Importe Diario
Total Salario Diario Integrado

\begin{tabular}{|c|c|c|c|c|}
\hline Trabajdor 1 & Trabajdor 2 & Trabajdor 3 & Trabajdor 4 & Trabajdor 5 \\
\hline 166.67 & 166.67 & 166.67 & 166.67 & 166.67 \\
\hline 6.85 & 6.85 & 6.85 & 6.85 & 6.85 \\
\hline 0.68 & 0.68 & 0.68 & 0.68 & 0.68 \\
\hline & & & & \\
\hline 11.67 & 8.33 & 10.83 & 10.00 & 6.67 \\
\hline & & & & \\
\hline 20.00 & 18.33 & 13.33 & 23.33 & 26.67 \\
\hline & & & & \\
\hline 26.67 & 36.67 & 32.50 & 25.00 & 29.67 \\
\hline & & & & \\
\hline 38.33 & 33.33 & 40.00 & 38.33 & 33.67 \\
\hline & & & & \\
\hline & & & & \\
\hline 100.00 & 100.00 & 100.00 & 100.00 & 100.00 \\
\hline & & & & \\
\hline 100.00 & 100.00 & 100.00 & 100.00 & 100.00 \\
\hline 470.87 & 470.87 & 470.87 & 470.87 & 470.87 \\
\hline
\end{tabular}

\section{Determinación del Salario Base de Cotización Art. 27 LSS}

\section{Concepto}

Salario Mensual $\$ 5,000.00$

Cuota Diaria

Aguinaldo 15 dias

Importe Diario

Prima Vacacional (Primer Año)

Importe Diario

Fondo de Ahorro Mensual $\$ 500.00$

(Cumple con los requistos del Art. 27 LSS)

Patrón

Despensa Mensual \$1,000.00

Importe Diario

Ayuda para Renta de Casa Mensual $\$ 3,000.00$

(Cumple con los requistos del Art. 27 LSS)

Importe Diario

Ayuda para Alimentación (3 Alimentos) $\$ 3,000.00$

(Cumple con los requistos del Art. 27 LSS)

Importe Diario

Premios de Puntualidad Mensual $\$ 550.00$

Importe Diario

Premios de Asistencia Mensual $\$ 1,100.00$

Importe Diario

\begin{tabular}{|c|c|c|c|c|}
\hline Trabajdor 1 & Trabajdor 2 & Trabajdor 3 & Trabajdor 4 & Trabajdor 5 \\
\hline 166.67 & 166.67 & 166.67 & 166.67 & 166.67 \\
\hline 6.85 & 6.85 & 6.85 & 6.85 & 6.85 \\
\hline & & & & \\
\hline 0.68 & 0.68 & 0.68 & 0.68 & 0.68 \\
\hline & & & & \\
\hline & & & & \\
\hline- & - & - & - & - \\
\hline 4.54 & - & 6.20 & 4.54 & - \\
\hline & & & & \\
\hline & & & & \\
\hline- & - & - & - & - \\
\hline & & & & \\
\hline & & & & \\
\hline- & - & - & - & - \\
\hline 2.13 & 0.91 & - & 5.46 & 9.25 \\
\hline & & & & \\
\hline 8.80 & 19.25 & 14.46 & 7.13 & 12.25 \\
\hline
\end{tabular}

\begin{tabular}{|c|c|c|c|c|c|c|c|c|c|c|}
\hline Total Salario Base de Cotización & $\$$ & 189.66 & $\$$ & 194.36 & $\$$ & 194.86 & $\$$ & 191.32 & $\$$ & 195.70 \\
\hline $\begin{array}{l}\text { Diferencia para el pago de prestaciones en dinero como consecuencia del } \\
\text { pago de prestaciones que se excluyen en terminos del Art. } 27 \text { LSS }\end{array}$ & $\$$ & 281.21 & $\$$ & 276.51 & $\$$ & 276.00 & $\$$ & 279.54 & $\$$ & 275.17 \\
\hline
\end{tabular}

Fuente: Elaboración propia (2019)

Tapia, E., Sánchez, S., Ordoñez, S.G. \& Lazcano, A.

El salario base de cotización y sus implicaciones en el pago de las prestaciones en dinero del seguro de riesgo de 149 trabajo 


\section{CONCLUSIONES}

1. El hecho de que el patrón incluya prestaciones excluyentes del salario base de cotización tiene como única finalidad el disminuir su carga tributaria en materia de aportaciones de seguridad social y no una afectación al trabajador.

2. Se concluye que el trabajador desconoce la disminución real que tendría respecto al pago de las prestaciones en dinero del seguro de riesgos de trabajo, en caso de que éste tuviera como consecuencia una incapacidad temporal, permanente parcial o total, o bien en su caso las derivadas de la muerte del asegurado, lo cual también implica una disminución real en las prestaciones que tengan derecho sus beneficiarios sin importar si se trata de pensión de viudez, orfandad o ascendientes.

3. Se considera que debe haber una separación de la base para el cumplimiento de la obligación patronal y la base para el pago de las prestaciones en dinero que recibe el trabajador, sin embargo, dicha separación pudiera repercutir en el patrimonio del Instituto Mexicano del Seguro Social ya que la base para determinar las cuotas obrero-patronales sería disminuir todas aquellas prestaciones que se consideran excluyentes de acuerdo al artículo 27 de la ley del seguro social, por su parte el Instituto pagaría las prestaciones correspondientes sobre una base mayor al no excluir las prestaciones del artículo antes citado.

\section{PROPUESTA}

Después de haber desarrollado la investigación y tomando en cuenta los resultados obtenidos, se considera que la mejor opción sería homologar las bases considerando todas las prestaciones que reciba el trabajador para el pago de cuotas y para el pago de las prestaciones en dinero, pero de manera que no se vean afectadas los intereses del patrón, del trabajador o bien en su caso del Instituto Mexicano del Seguro Social, estableciendo una base justa y equitativa para los tres sujetos involucrados.

\section{REFERENCIAS}

Cruz, P. D. (2014). Aspectos juridicos y contables de la Ley del Seguro Social. México: Ediciones Fiscales ISEF.

Tapia, E., Sánchez, S., Ordoñez, S.G. \& Lazcano, A.

El salario base de cotización y sus implicaciones en el pago de las prestaciones en dinero del seguro de riesgo de 150 trabajo 
Gutierrez, F. J. (2018). Mi pension. Requisitos y cálculos que debo conocer para obtenerla 2018. México: Ediciones Fiscales ISEF.

Gutierrez, J. M. (2018). El ABC Fiscal de sueldos y salarios 2018. México: Ediciones Fiscales ISEF.

IMSS. (2018). Instituto Mexicano del Seguro Social . Obtenido de http://www.imss.gob.mx/

Olguín, J. P. (2014). Manual de Aplicación y Casos Practicos de Seguridad Social. México: Tax Editores.

Olguín, J. P. (2017). Ley Federal del Trabajo 2017.Analisis y comentarios 2017. México: Tax Editores.

Olguín, J. P. (2017). Manual para el Control Integral de las Nóminas 2017. México: Tax Editores.

Olguín, J. P. (2017). Taller de Prácticas Laborales y de Seguridad Social 2017. México: Tax Editores.

Públicos, I. M. (2015). Acuerdos Relevantes del Consejo Tecnico del IMSS. México: Instituto Mexicano de Contadores Públicos.

Públicos, I. M. (2017). Estudio Integral de las pensiones que otroga el IMSS. México: Instituto Mexicano de Contadores Públicos.

RLSSMACERF. (15 de Julio de 2005). Reglamento de la Ley del Seguro Social en Materia de Afiliación, Clasificación de Empresas, Recaudación y Fiscalización. Obtenido de http://www.diputados.gob.mx/LeyesBiblio/regley/Reg LSS MACERF.pdf

RSSOTCOTD. (04 de Marzo de 2008). Reglamento del Seguro Social Obligatorio para los Trabajadores de la Construccón por Obra o Tiempo Determinado. Obtenido de http://www.imss.gob.mx/sities/all/statics/pdf/reglamentos/4044.pdf

Social, C. r. (2008). Pensiones.Estudio integral de pensiones que otorga el IMSS. México DF: Instituo Mexicano de Contadores Públicos. 